\title{
Téoros
}

Revue de recherche en tourisme

\section{Sites historiques, musées de plein air et parcs thématiques : une recette américaine}

\section{Raymond Montpetit}

Volume 12, numéro 3, octobre 1993

Le renouveau des parcs à thèmes

URI : https://id.erudit.org/iderudit/1077935ar

DOI : https://doi.org/10.7202/1077935ar

Aller au sommaire du numéro

Éditeur(s)

Université du Québec à Montréal

ISSN

0712-8657 (imprimé)

1923-2705 (numérique)

Découvrir la revue

Citer cet article

Montpetit, R. (1993). Sites historiques, musées de plein air et parcs thématiques : une recette américaine. Téoros, 12(3), 26-29.

https://doi.org/10.7202/1077935ar d'utilisation que vous pouvez consulter en ligne. 


\title{
Sites historiques, musées de plein air et parcs thématiques : une recette américaine
}

\author{
Raymond Montpetit*
}

Nous ne saurions surestimer l'impact du tourisme etdes lieux qui réussissent auprès des touristes, sur l'évolution de la muséologie de l'histoire; parce que les clientèles touristiques comptent de plus en plus pour les musées qui présentent l'histoire de leur ville, de leur région ou de leur pays, ces institutions sont plus conscientes que jamais de s'adresser à des gens qui arrivent avec très peu de connaissances préalables de cette histoire, mais avec souvent une bonne expérience de la visite de musées et de d'autres sites touristiques qui ont retenu leur attention ailleurs.

Nous voudrions dans ce bref article, examiner un peu les liens et les similitudes qui unissent la muséologie de l'histoire qui s'est développée aux États-Unis, le pays d'origine des parcs thématiques au sens contemporain de cette expression.

\section{Les parcs et les musées}

Depuis longtemps, les parcs et les musées entretiennent des relations de proche parenté. Cela tient à leur réalité même: dans les deux cas, il s'agit bien de lieux tridimensionnels de regroupements et de représentations. Ces espaces structurent et mettent en place des parcours définis parmi des choses exposées, cela pour le plaisir de ceux qui y déambulent à la découverte des perspectives que ces endroits aménagent et des spécimens choisis - naturels et culturels, qu'ils contiennent et font voir.

Au parc et au musée, nous sommes devant des espaces planifiés, devant des lieux qui instaurent une logique bien à eux, hors de l'espace commun de la vie de tous les jours et qui ouvre, dans le quotidien, une dimension autre. Dans ces environnements contrôlés, le visiteur est pris en charge, et tout ce qui lui est donné à percevoir l'est en fonction d'une straté-

Monsieur Raymond Montpetit est directeur de la maitrise en musbologie a l'Université du Quebec à Montrésal.

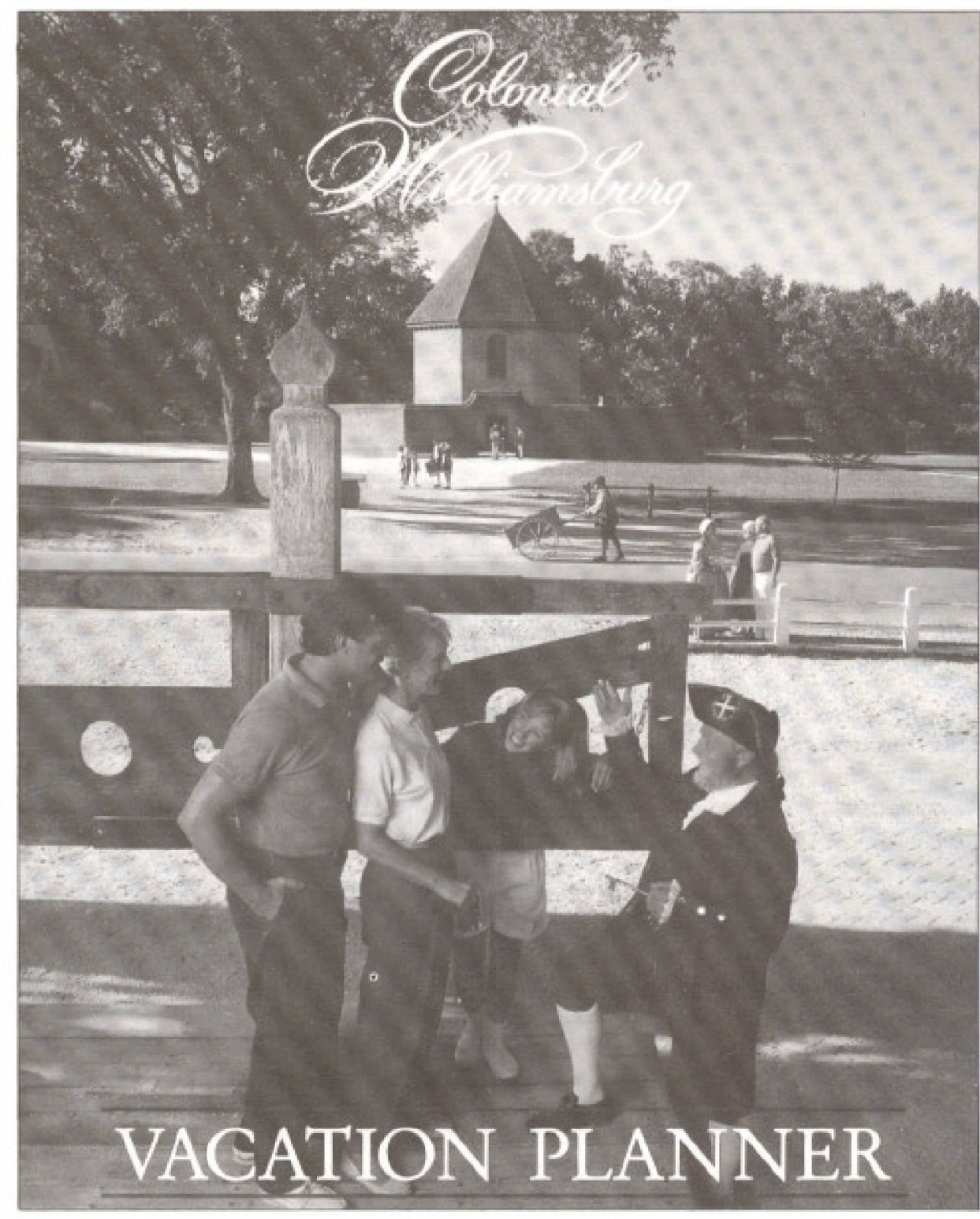

gie visuelle particulière. L'espace est réglé pour produire principalement des *effets de plaisirs, cela au moins à deux niveaux, à celui de l'ensemble perçu, lespace déployé, et à celui des chares observéer, la collection rassemblée.

Au niveau le plus global, celui de l'espace, un «effet de plaisir», émane de la contemplation et de la déambulation dans un environnement agréable programmé en fonction de ceux qui s'y rendent.
L'espace d'exposition - musée ou parc a certes changé avec les époques, son design a évolué, mais il a toujours constitué un lieu bien particulier qui physiquement se distingue de tout autre. L'amateur prend plaisir à être là, à se promener en ces lieux denses, à parcourir, physiquement et du regard, les zones closes successives et les points de vue ouver ts sur le lointain; il aime se livrer aux sensations nouvelles et changeantes que musée et parc lui offrent en tant qu'espa- 
ces de découvertes où des réalités sont disposées à son intention.

À un niveau plus microscopique, celui de la collection, lobservation plus en détail des différents éléments exposés, oeuvres ou spécimens, procure aussi des effets de plaisir qui sont davantage reliés cette fois aux connaissances que le visiteur a des choses qu'il voit. Le botaniste jouit de la présence de telle plante rare et exotique et note ses caractéristiques; l'amateur de peinture examine le traitement des couleurs et de la perspective dans un tableau de maître. Ainsi, plaisir de l'ambiance (contemplation/déambulation) et plaisir des choses (observation/connaissance) se conjuguent pour *intéresser tout à la fois l'oeil et l'esprits (I) et assurer le caractère attrayant tant du musếe que du parc.

Tout au long du XIXe sitcle, en Europe comme en Amérique, nous connaissons le musée, qui étale des artefacts dans des salles selon des principes de classification comme la «chronologies, «l'école artistiques ou la *provenances, le jardin des plantes, qui dispose ses spécimens en relation avec les catégories d'analyse des sciences naturelles, et le parc qui montre ses collections selon des règles de présentation qui imitentet suggèrent l'apparence naturelle du monde ambiant. Cet ordre de choses domine jusqu'aux dernières décennies du XIXe sièle, quand le parc et le musée, jusqu'à un certain point, fusionnent, pour faire qu'apparaissent les premiers *musées en plein airs; ceux-ci reprennent pour ainsi dire, la forme du parc, mais pour y rassembler et $y$ montrer autre chose que des spécimens naturels.

\section{Les maisons et les sites historiques}

La première forme de «parc historique* s'implantelorsquele mouvement naissant de conservation se propose, vers 1850 , de sacraliser certains sites; ainsi, les maisons où vécurent de grands personnages et les lieux où, comme on a pris l'habitude de le dire, \&l'histoire s'est écrites, deviennent des «sites historiques» que l'on cherche à conserver pour commémorer ceux qui $y$ ont vécu et les événements marquants qui s'y sont déroulés. Aux États-Unis, il est assez compréhensible qu'au moment où disparaissent les derniers représentants de la génération qui a vécu l'époque de la révolution et de la fondation de la République, certains ama teurs d'histoire son- gent à préserver les lieux qui témoigneront de ces événements aux yeux des générations futures. C'est ainsi qu'en 1850 , l'on sauve Hasbrouck House, le quartier général de George Washington à Newburgh; puis en 1858 , Mount Vernon, son domaine en Virginie est acquis par le «Mount Vernon Ladies' Associations qui veut *conserver avec une révérence sacrée et en l'état où Washington les laissa», sa maison et les terrains qui l'entourent. Plus tard Monticello, propriété de Thomas Jefferson, est aussi transformée en wsite historique*. Dans tous ces lieux, on peut se rendre comme à l'église, afin d'accomplir un pèlerinage en l'honneur de ces pères fondateurs, selon le rituel d'une nouvelle religion civile et patriotique centrée sur ces personnages.

La création de ces lieux historiques concurrence les musées d'histoire plus traditionnels déjà en place; comme les musées d'art qui exposent les oeuvres, ou comme les musées d'archéologie qui exhibent les fragments de civilisations souvent disparues, les musées d'histoire collectionnent et exposent, le plus souvent en vitrines, des objets anciens coupés de leurs contextes. Or, les nouveaux lieux historiques s'avèrent être porteurs à la fois de plus d'émotion et de plus de véracité. Le visi= teur entre, pour ainsi dire, dans un environnement qu'il ressent comme étant de toute part relié à l'histoire, il marche là où l'histoire s'est faite et devient lui-même un «quasi-témoin*, capable de visualiser une époque qui n'est plus mais qui pourtant, perdure encore ici, dans un contexte qui paraît avoir échappé au passage du temps.

Il apparaît plus clairement que l'histoire ne saurait s'incarner uniquement dans une collection d'objets; son caractère de récit exige une narration qui visuellement, prenne appui sur plusieurs ordres de réalité, sur $1^{\circ}$ des objets bien sûr, mais aussi sur $2^{\circ}$ des idées, $3^{\circ}$ des actions, $4^{\circ}$ des personnes et sur $5^{\circ}$ des réseaux de relations qui composent les ensembles contextuels où les faits de l'histoire ont pris place.

Le site historique cherche à déployer concrètement l'univers dans lequel l'histoires'est produite, il traduit la cohếrence du tout qui donne sens aux personnages et aux événements de la thématique particulière qui est la sienne. Aussi, maisons et sites historiques préservés nous paraissent-ils constituer les premiers «pares thématiques»; là, objets, architectures, environnements et bientôt animations et théa tralisations, se conjuguent en un espace thématique dont le réalisme interpelle tous les sens du visiteur afin de le plonger dans une temporalité et une histoire autres.

\section{Les musếes de plein air}

L'histoire des musếes retient le nom d'Arthur Hazelius comme celui qui créa en Suède, le premier musée de plein air; mais nous dirions que c'est aux ÉtatsUnis que cette formule s'est le plus développée, sous l'impulsion d'une relation réciproque et dynamique entre musée de plein air et parc thématique. Les musées de plein air ont connu aux Etats-Unis un important développement qui a conduit à la création d'une multitude d'établissements où les collections historiques sont mises en exposition hors les murs d'un musée, à l'aide d'une muséographie qui utilise plusieurs formes d'animations, de reconstitutions, de restaurations et de simulations, pour faire revivre une thématique historique au visiteur.

Un musée de plein air peut se définir comme la reconstitution d'un enclave historique, comme

\section{[.+. ] a full-scale duplication, insofar as passible, of place, a point in time and a way of life, the main purpose of which is to communicate broadly to the visitor the experiences of that segment of the past. Participation by the visitor in these experiences, limited though they may be, will tend to deepen bis perceptions and provide bim witb a richer understanding of bis own time and person. (2)}

Les musées de plein air comptent donc plusieurs bâtiments qui datent d'une période antérieure au présent, avec à l'intérieur le mobilier et les équipements appropriés, le tout dans un environnement aussi conforme avec celui de l'époque retenue. Ces enclaves peuvent ou bien mettre en valeur un site ancien réel, documenté et présenté tel qu'il a réellement existế, ou bien illustrer un prototype, en aménageant un site qui soit typique et représentatif d'une certaine réalité passée, comme un village régional, une ferme 
Expériences

Environnement

Cinéma

Spectacle

Gastronomie

Marché

Artefacts

Colonial Williamsburg

Plimoth Plantation

Restauration et reconstruction

sur l'emplacement véritable

Film introductif dans le

*Visitors Center*

Animation par des interprètes en costumes d'époque.

Démonstrations de métiers

Présence sur le site de plusieurs auberges anciennes offrant des menus d'époque

Présence sur le site de boutiques d'artisanat

Nombreux artefacts sur le site
Reconstruction d'une wcopie* sur site autre

Multimédia introductif au pavillon d'accueil

Theatralisation at la première personne: par interprètes/acteurs

Tenue d'événements spéciaux avec repas façon XVIle siècle

Deux boutiques

cadeau

Collection de référence exposéc au pavillon typiquedes établissementsagricoles d'une période, etc. Les premiers, véritables musées de sité, émanent du lieu et illustrent šur place les événements er les gens qui ont fait l'histoire en cet endroit; les seconds, sans lien avec leur emplacement actuel, regroupent en un lieu commode et propice, les éléments d'une collection qui composent un environnement réaliste et vraisemblable pour les thèmes historiques qu'ils se proposent de traiter et les collections d'objets qu'ils exposent.

Le musée de plein air est donc d'abord une ré́alitế environnementale; en disposant ses artefacts grandeur nature dans l'espace selon une ordonnance qui ellemême relève d'une configuration historique, il maximise l'historicité des bâtiments et confere au paysage tout entier qu'il instaure, lestatut de témoin du passé.

Dans ce type de musée, on peut dire que le contenant fait lui-même partie du contenu, les deux se rejoignant pour dire l'histoire et permettrel'entrée en sa réalité paysagée. Ces musées agissent comme des parcs thématiques historiques, *they encourage us to wander among their recreated or reconstructed premisses entertainingly, instructively, and perhaps thought-provokingly, out of time.s $(m)$

Reprenant ses formes, faisant woir ses objets, le passé paraît aussi pouvoir reprendre vie. Cest pourquoi plusieurs musées de plein air américains optent en plus pour les méthodes d'animation et d'interprétation dites de aliving historys. ou *d'histoire vivantes, en ajoutant dans leurs espaces, des représentations de personnages grâce à des rôles confiés à des acteurs/interprètes; pour mieux évoquer le passé, pour augmenter l'effet de réalitế, ils complètent ainsi les décors et les objets de l'histoire par la reconstitution des personnes qui peuplaient le lieu et celle de leurs actions.

\section{Les parcs thématiques}

Les parcs thématiques créent aussi des espaces qui incarnent et matérialisent des images et des thèmes le plus souvent empruntés aux récits dêjầ médiatisés de la culture populaire en circulation. Ceux de Walt Disney, par exemple, prêtent vie à des mythologies *maison $*$, diffusées dès l'enfance par plusieurs autres médias. Ils permettent d'entrer un moment dans ces mondes fictionnels, de faire *comme sis ces mythologies avaient vraiment lieu et de séjourner auprès de ce qui se donne comme la source réelle, wen chair et en $0 s \%$, de toute cette imagerie célèbre. Il est intéressant d'ailleurs de noter que ce faisant, ces pares thématiques génèrent de vrais objets de collection, des artefacts datés, qui trouvent un marché bien organisé de collectionneurs qui paient un prix qui va croissant avec la rareté et la demande.
Les définitions du parc thématique contemporain sont nombreuses et, à leur lecture, on constate les similitudes manifestes entre ces parcs et les musées historiques de plein air.

A tbeme park is an extensive
commercial leisure-park develop-
ment wbich normally includes
several tbemed areas or indoor and
outdoor displays, activities,
amusements and fully fledged
animation, plus well developed
catering, retailing and visitor
services. Cbaracteristictbemesmay
be bistorical, geograpbical, social, or
relate to a futuresociety, or to future
in industry.

Parce que le musée de plein air est un lieu qui cherche à donner forme et présence à des réalités et des figures historiques connues du grand public, il poursuit des objectifs analogues à ceux des pares thêmatiques plus axés sur des scénarios aux références mythologiques et fictionnelles; de même, il utilise des procédés et des techniques qui ressemblent à ceux en vigueur dans les parcs thématiques. Les musées historiques de plein air font aussi *comme si pénétrer dans les lieux réels de l'histoire était entré dans le passé, *comme si ces vestiges devaient tout au passé et rien aux interventions du présent, *comme siw changer d'environnement était changer de temps. L'effet provoqué en est un où la temporalité des 
choses exposées se substitue à la réalité temporelle du visiteur; celui-ci, entouré d'un environnement peu familier et attribué au passé, en vient à consentir à se croire déplacé dans le temps, alors qu'il ne l'est que dans l'espace.

Certes, les parcs thématiques sont des établissements à but lucratif et cela les distinguent bien des musées; mais les coûts importants qui incombent aux musées de plein air exigent aussi de leur part des efforts concertés de commercialisation dont les fruits sont cependant réinvestis dans l'opération et le développement de l'institution.

\section{Colonial Williamsburg et Plimoth Plantation}

Deux musées historiques de plein air Colonial Williamsburg en Virginie et Plimoth Plantation au Massachusetts, ont a la fois pu inspirer les premiers parcs thématiques nés à partir des années 1950, et par la suite, emprunter des méthodes et des techniques d'animation développées dans ces nouveaux parcs thématiques.

Durant les années 1920-1930, le riche financier américain John D. Rockefeller $\mathrm{Jr}$. achète, fait restaurer et aussi reconstruire de toutes pièces, la petite ville de Williamsburg, fondée en 1633 et capitale coloniale de 1699 à 1780 . Tout ce qui date d'après 1790 est démoli, dans le but de redonner à l'ensemble l'apparence qui prévalait durant la période $1770-1780$, cela en conformité avec un plan de ville daté de 1782. En 1932, quelques années seulement après l'ouverture du site au public, les responsables ont recours à des guides en costumes d'époque, qui expliquent l'histoire et l'architecture aux visiteurs et font des démonstrations ayant trait aux métiers traditionnels. À partir des années 1970, Williamsburg attireplus d'un million de visiteurs par an et promet à ceux qui $s^{\prime} y$ rendent, une expérience inoubliable avec des slogans comme *Venez et entrez dans le XVIIIe siècls $\%$ *Revivez la naissance de la nations. A Williamsburg, tout l'environnement et les collections qui y logent, suggèrent un voyage dans l'histoire, à la rencontre de Washington, des premiers révolutionnaires, des dirigeants de la République et des autres citoyens anonymes.

Le musée en plein air de Plimoth commence plus simplement: en 1945, un ri- chehommed'affaires, Henry Hornblower II, crée une société dans le but «de créer, de construire et de maintenir un village de $\approx$ Pilgrims $*$, afin de commémorer les premiers «Pilgrims Fathers» arrivés à Plimoth en $1620 m$. Il s'agit dans ce cas, de construire une «copies du village complètement disparu, sur un emplacement qui se trouve à quelques kilomètres du site réel du premier établissement des fondateurs de Plimoth. En 1957, un navire, réplique approximative du Mayflower sur lequel les premiers *Pilgrims* sont arrivés en 1620, est installé et ouvert aux visiteurs, et en 1959 , le musée de plein air avecses maisons reconstituées et ses guides en costumes, connaît une première saison régulière.

Depuis les années 1970 , le musée de Plimoth Plantation a pousséplus loin que tout autre l'expérience du \&living history». Les acteurs/interprètes parlenten Anglais du XVIIe siècle avec un accent qui correspond à leur région de provenance; ils parlent aussi à première personne, tenant ainsi comme au thêatre le rôle d'un personnage réel, dont ils adoptent le point de vue etl'identité. De plus, en répondant aux questions que peuvent leur poser les visiteurs, ils feignent d'ignorer tout ce qui a eu lieu depuis 1627 , année choisie comme année de référence des activités et événements reconstitués au village. Ces personnages vaquent à leurs occupations quotidiennes dans le village reconstitué, conformément à ce que l'on sait des travaux et manières de la période 1620 et en utilisant les outils et les techniques de ce temps. Tout au village, objets, architectures, costumes, est le fruit d'un travail important de documentation et de reproduction, aucun objet authentiquement ancien n'y figure. Aussi les visiteurs peuvent-ils toucher à tout, entrer dans les maisons, utiliser le mobilier qu'elles contiennent, tout cela dans une expérience d'immersion totale et très impressionnante dans le passé.

Expérience est ici le mot clef; pour ces musées, «revivre» semble remplacer l'ancien *faire voir et savoir» qui prévalaitdans les expositions en salle. Analysant le fonctionnement des pavillons d'Epcot, George Macdonald note que leur formule repose sur la combinaison de différentes expériences offertes au visiteur: expérience environnementale, cinématographique, du spectacle, gastronomique, de la place du marché et expérience del'artefact(?). Cesmêmes expériences se conjuguent aussi dans la plupart des musées historiques de plein air.

Les musées historiques de plein air veulent, à la manière des parcs thématiques, offrir au visiteur plus qu'une collection d'objets à contempler. Ils promettent une expérience unique pluridimensionnelle, qui donne l'impression d'échapper aux contraintes du réel pour livrer accès à un autre temps. Ils réalisent cet exploit au moyen d'efforts planifiés et ingénieux pour installer un espace fictionnel très contrôlé et vraisemblable; cet espace revêt l'apparence des vestiges authentiques d'une autre époque en prenant appui sur une recherche documentaire et sur des objets de collection.

Comme au théâtre, au cinéma ou au parc thématique, levisiteur de ces musées joue le jeu, il accepte de *participer $\%$, il imagine et se laisseprendre aux ruses de la mise en scène de l'histoire. Des lieux où se montre ce qui n'est plus. Des lieux où se voit ce qui n'est qu'imagination, «Et sion jouait au passé.... Allons au musée de plein air:» $f$

\section{Notase}

(1) Rent-Louls de Girardin, De la composition des payanges, 1777, rétedition Champ Vallon, 1992. ค. 221.

(2) Wavne Colwell. Windows on the past, in Museum News, vol. 50, no 10, juin 1972, pp. 36-37. Selon une definition adoptée par le Conseil international des muśes (ICOM)en 1957, le musée de plein air est *une collection d'objets historiques a) qui est ouverte au public; b) qui comprend des spécimens d'architecture de style populaire de l'époque préindustrielle thabitations rurales et urbaines, ateliers et batiment annexes; et c) qui comprend des cheifs-d'oeurre d'architecture tels que des grandes demeures, des églises ou des batiments historiques de l'époque industrielles. Voir aussi Edward P.Alexander, Museum Masters, A.ASLH, Nashville, 1993, qui reprend cette definition p. 267.

(3) Colin Soronsen, theme Parks and time Machines, in the New Museology. Reaktion Books, Londres, 1989, pp. 61-62.

(4) A.S. Travis, Museums, Theme-Parks and Developments in Tourism, in Loisir er socibtes, vol. 10. no 1 , printemps 1987, P. 16. Voir aussi Patricia Mackay, theme Parks US.A, in Theater Crafts, eptembre 1977, qui deffinit le parc thématique comme sa familly entertainment complex oriented to a particular subject or historical area, combining the continuity of costuming and architecture with entertainment and merchandise to create a fantasy-provoking atmospheres, p. 56.

(5) George Macdonald, Epcot Center vu dans une perspecthe musdale, in Muse, printempa 1988, pp. 33-34. 